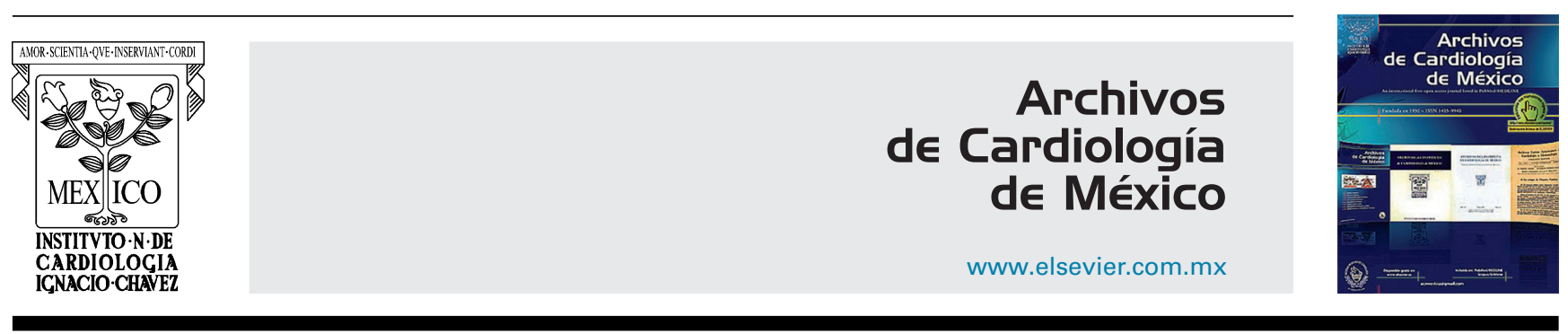

ARTÍCULO DE REVISIÓN

\title{
Arco aórtico derecho con subclavia izquierda aberrante y divertículo de Kommerell. Una causa de anillo vascular
}

\section{Tania Tamayo-Espinosa*, Julio Erdmenger-Orellana, Rosario Becerra-Becerra, Norma Balderrabano-Saucedo y Begoña Segura-Standford}

Departamento de Cardiología, Hospital Infantil de México Federico Gómez, Ciudad de México, México

Recibido el 23 de mayo de 2016; aceptado el 28 de marzo de 2017

\section{PALABRAS CLAVE \\ Divertículo de Kommerell; Arco aórtico derecho; Anillo vascular; Subclavia izquierda aberrante; México}

\section{KEYWORDS}

Kommerell's diverticulum; Right aortic arch; Vascular ring; Aberrant left subclavian artery; Mexico
Resumen El arco aórtico derecho puede estar asociado a subclavia izquierda aberrante, en algunos casos esta se origina de una dilatación aneurismática que se conoce como divertículo de Kommerell.

Se presentan 2 casos de anillo vascular formado por un arco aórtico derecho, subclavia izquierda anómala con divertículo de Kommerell y persistencia del conducto arterioso izquierdo con una revisión de la literatura acerca del desarrollo embriológico y los métodos de imagen que ayudan al diagnóstico de esta rara anomalía vascular.

(c) 2017 Instituto Nacional de Cardiología Ignacio Chávez. Publicado por Masson Doyma México S.A. Este es un artículo Open Access bajo la licencia CC BY-NC-ND (http://creativecommons. org/licenses/by-nc-nd/4.0/).

Right-side aortic arch with aberrant left subclavian artery and Kommerell's diverticulum. A cause of vascular ring

\begin{abstract}
The right-side aortic arch may be associated with aberrant left subclavian artery, in some cases this artery originates from an aneurismal dilation of the aorta called Kommerell's diverticulum. A report is presented on 2 cases of vascular ring formed by a right-side aortic arch, anomalous left subclavian artery, Kommerell's diverticulum and left patent ductus arteriosus. A review the literature was also performed as regards the embryological development and the imaging methods used to help in the diagnosis of this rare vascular anomaly.

(c) 2017 Instituto Nacional de Cardiología Ignacio Chávez. Published by Masson Doyma México S.A. This is an open access article under the CC BY-NC-ND license (http://creativecommons. org/licenses/by-nc-nd/4.0/).
\end{abstract}

\footnotetext{
* Autor para correspondencia. Dr. Márquez 162, Col. Doctores, Delegación Cuauhtémoc, Ciudad de México, C.P 06720. Teléfono: +52289917; extensión 2448; fax: +56718001 .

Correo electrónico: taniatamayoespinosa@gmail.com (T. Tamayo-Espinosa).
} 


\section{Introducción}

EL arco aórtico derecho es una cardiopatía que ocurre en el 0.05-0.1\% de la población general. De acuerdo a la emergencia de los vasos supraaórticos, existen 3 tipos de arco derecho: tipo ।: con vasos supraaórticos en espejo, tipo II: con subclavia izquierda aberrante y tipo ॥I: con origen de la subclavia izquierda en la arteria pulmonar ${ }^{1}$. Cuando se asocia a una subclavia izquierda aberrante, esta arteria es la última en originarse del arco aórtico y atraviesa el mediastino de derecha a izquierda con 3 diferentes trayectos: posterior al esófago (80\%), entre el esófago y la tráquea $(15 \%)$, o anterior a la tráquea $(5 \%)^{2}$. En algunos casos la arteria subclavia izquierda se origina de una dilatación aneurismática conocida como divertículo de Kommerell el cual es una rara anomalía congénita que puede ocasionar por sí mismo compresión traqueoesofágica ${ }^{3}$ y existen reportes de riesgo de rotura y disección ${ }^{4}$.

\section{Reporte de casos}

Caso 1. Masculino de 2 meses de edad. Se inició estudio por falla de medro y soplo cardiaco. La exploración física, radiografía de tórax y electrocardiograma indicaban persistencia del conducto arterioso (PCA). En el ecocardiograma se observó dilatación de cavidades izquierdas, arco aórtico derecho y persistencia de conducto arterioso izquierdo. Por lo anterior se sospechó anillo vascular y se solicitó angiotomografía en la cual se observó: arco aórtico derecho con subclavia izquierda aberrante la cual se origina de una dilatación aneurismática de la aorta dorsal distal y persistencia del conducto arterioso hacia la rama izquierda de la arteria pulmonar; estas estructuras vasculares rodean la tráquea y el esófago formando un anillo vascular completo (fig. 1).

Caso 2. Masculino de 2 meses de edad, referido por insuficiencia cardiaca, clínicamente con sospecha de conducto arterioso permeable. En el ecocardiograma se observó: ventrículo izquierdo no compactado, dilatación aneurismática del tronco de la arteria pulmonar, arco aórtico derecho y conducto arterioso izquierdo. La angiotomografía corroboró los hallazgos y demostró: anillo vascular formado por el arco aórtico derecho, subclavia izquierda aberrante originándose de un divertículo de Kommerell y persistencia del conducto arterioso izquierdo, así como ventrículo izquierdo no compactado y dilatación aneurismática de la arteria pulmonar (figs. 2 y 3 ).

A los 2 pacientes se les realizó sección y sutura del conducto arterioso dejando la subclavia izquierda con abordaje por toracotomía izquierda.

\section{Discusión}

El divertículo de Kommerell se ha asociado a arco aórtico derecho con ligamento o conducto arterioso izquierdo formando un anillo vascular anatómico completo que causa compresión traqueosofágica ${ }^{5}$. De acuerdo al estudio de Backer et al., es la segunda causa de anillo vascular, después del doble arco aórtico ${ }^{6}$. La prevalencia del arco aórtico derecho con subclavia izquierda aberrante se ha reportado en el $0.04-0.4 \%$ de los cuales el $20-60 \%$ puede asociarse a divertículo de Kommerell ${ }^{6-8}$.

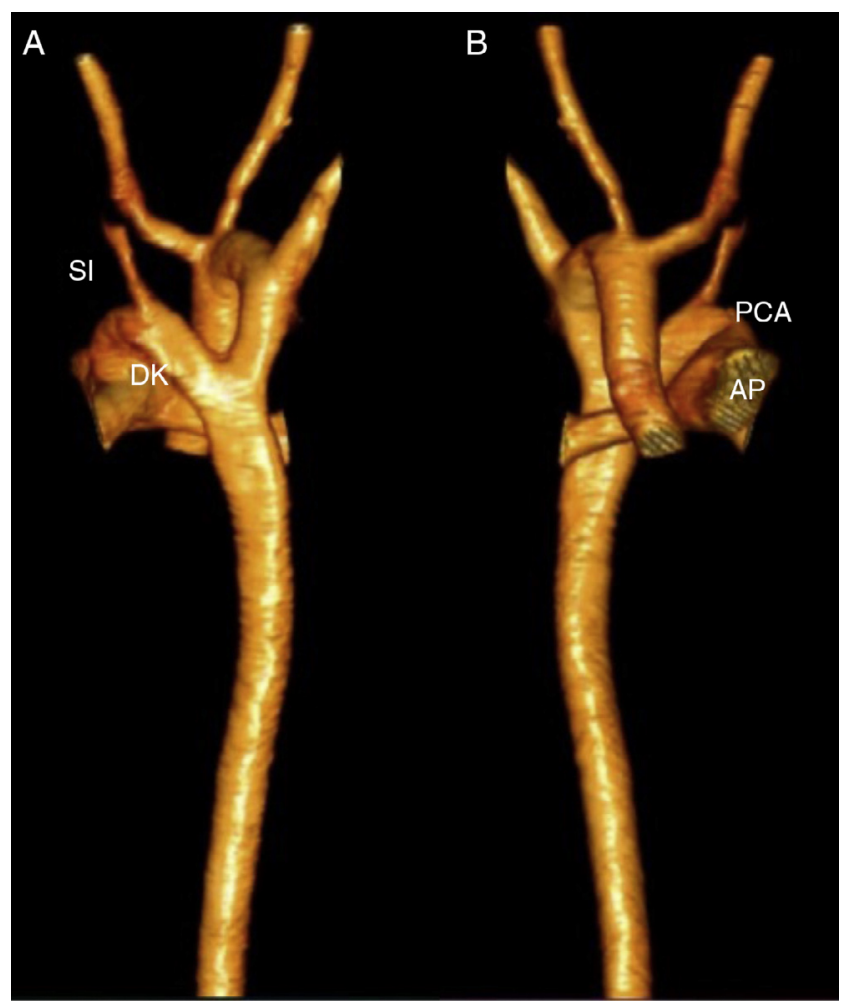

Figura 1 A) Reconstrucción tridimensional por angiotomografía; vista anterior del arco aórtico derecho, la arteria pulmonar (AP) y su continuidad con el conducto arterioso (PCA). B) Vista posterior del divertículo de Kommerell (DK) del cual se origina la arteria subclavia izquierda (SI).

Esta anomalía vascular fue descrita en 1936 por el radiólogo alemán Buckhard F. Kommerell en un paciente adulto con arco aórtico izquierdo; durante un examen con trago de bario demostró que a la izquierda y detrás del esófago había una masa pulsátil que lo empujaba hacia adelante a nivel del botón aórtico. En su publicación original escribió: «la masa pulsátil detrás del esófago no corresponde a la arteria subclavia derecha aberrante debido a que el calibre de este vaso es mucho más pequeño. Es más probable que esta masa corresponda a un divertículo aórtico, de la cual se origina la arteria subclavia derecha... ${ }^{9}$.

Esta anomalía ocurre durante la cuarta y quinta semana de la vida fetal cuando existen 6 pares de arcos aórticos conectados a la aorta ventral y dorsal. Normalmente el cuarto arco aórtico izquierdo persiste y se continúa con la aorta ascendente y descendente para formar el arco aórtico izquierdo normal. El arco aórtico derecho ocurre cuando el cuarto arco derecho persiste y se continúa con la aorta dorsal derecha y el cuarto arco izquierdo involuciona junto con la aorta dorsal izquierda ${ }^{1}$. La arteria subclavia izquierda aberrante es el resultado de la regresión del cuarto arco aórtico izquierdo entre la carótida izquierda y la arteria subclavia izquierda9. El divertículo de Kommerell ocurre por la persistencia de la porción distal del cuarto arco aórtico, que en estos casos es el izquierdo ${ }^{10}$. Generalmente el divertículo esta bien desarrollado, debido a la gran cantidad de volumen sanguíneo que recibe del conducto arterioso fetal ${ }^{11}$. 


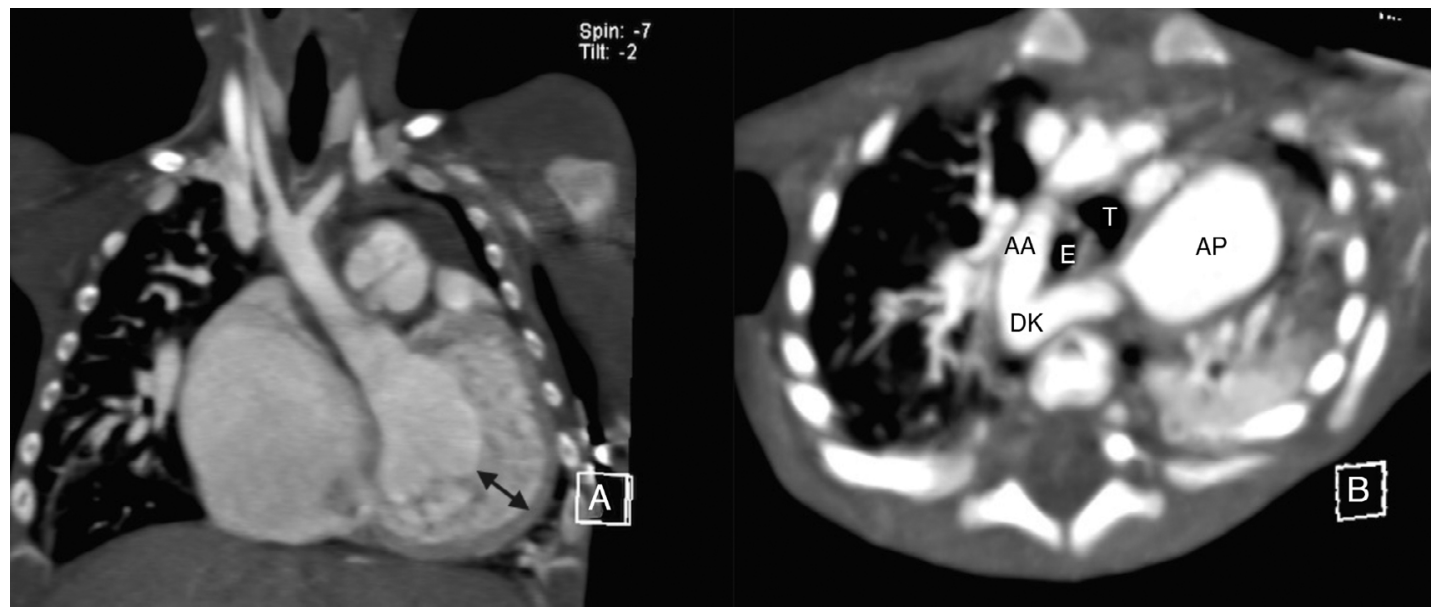

Figura 2 A) Angiotomografía; vista coronal del ventrículo izquierdo no compactado señalado entre flechas. B) Imagen axial que muestra la relación de arco derecho (AA), divertículo de Kommerell (DK) y arteria pulmonar (AP) rodeando tráquea (T) y esófago $(\mathrm{E})$.

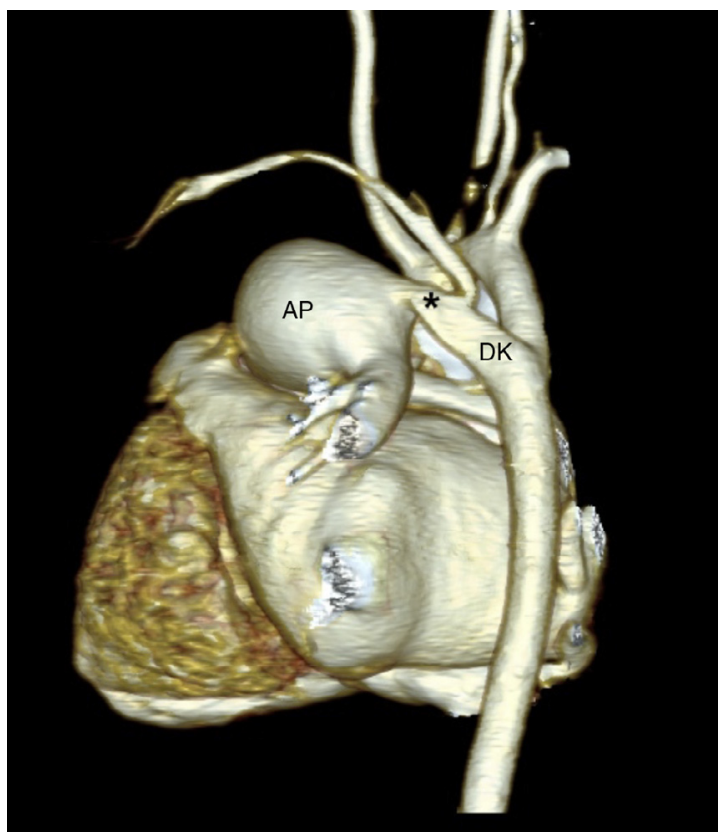

Figura 3 Angiotomografía con reconstrucción tridimensional que muestra la dilatación aneurismática de la arteria pulmonar (AP), el PCA $\left({ }^{*}\right)$, y el nacimiento de la arteria subclavia izquierda a partir del divertículo de Kommerell (DK).

El paciente con anillo vascular completo puede presentar síntomas respiratorios y digestivos que dependen del grado de compresión que generan las estructuras vasculares sobre la tráquea o el esófago. En la edad pediátrica predominan los síntomas respiratorios como estridor que se exacerba con la alimentación, tos crónica, infecciones recurrentes de vías respiratorias, episodios de cianosis o disnea y en el paciente mayor puede aparecer disfagia a sólidos ${ }^{6}$.

Los anillos vasculares pueden observarse de forma indirecta en la radiografía de tórax o en el esofagograma con bario; en la radiografía de tórax anteroposterior puede verse la posición del arco aórtico por la desviación contralateral de la tráquea y la compresión traqueal se demuestra en la radiografía lateral, de manera que encontrar un arco aórtico derecho con compresión traqueal aumenta la posibilidad diagnóstica de anillo vascular. En el esofagograma con bario se puede observar la impresión de la estructura vascular sobre la pared posterior del esófago ${ }^{12}$.

El ecocardiograma está indicado para el estudio de cardiopatías asociadas; Backer et al. reportaron la asociación con comunicación interventricular, tetralogía de Fallot, PCA, transposición corregida de grandes arterias, ausencia de rama izquierda y dextrocardia ${ }^{6}$. En las vistas supraesternales en eje corto con angulación anterior se puede observar la aorta transversa y la emergencia del primer vaso supraaórtico, si este se dirige hacia la izquierda indica que el arco aórtico es derecho; al seguir el trayecto de este vaso, el no observar su bifurcación muestra la presencia de una subclavia izquierda aberrante la cual puede tener un curso retroesofágico formando el anillo vascular en presencia de un conducto arterioso o ligamento arterioso. En nuestros casos, la importante repercusión hemodinámica que ocasionaba el conducto arterioso fue motivo del estudio y el hallazgo de un arco aórtico derecho asociado a un conducto arterioso relacionado con la arteria pulmonar izquierda despertó la sospecha diagnóstica (fig. 4).

La tomografía computada demuestra con detalle las características anatómicas que permiten planear el abordaje y el tipo de corrección quirúrgica; y se considera el estudio de elección en el paciente con anillo vascular ${ }^{6,13}$.

El diagnóstico diferencial debe hacerse con el doble arco aórtico; la diferencia es que el divertículo es un remanente del arco dorsal sin continuidad con la aorta ascendente ${ }^{10}$.

El tratamiento va dirigido a la liberación quirúrgica del anillo vascular, existe controversia sobre cuál es la mejor técnica. Backer et al. reportaron 8 pacientes con síntomas recurrentes después de la resección del ligamento arterioso que requirieron una segunda intervención quirúrgica para resecar el divertículo después de lo cual los síntomas desaparecieron, por lo que recomiendan como primera elección en este tipo de anillo vascular la resección del divertículo y el reimplante de la arteria subclavia izquierda a la carótida izquierda ${ }^{14}$. 


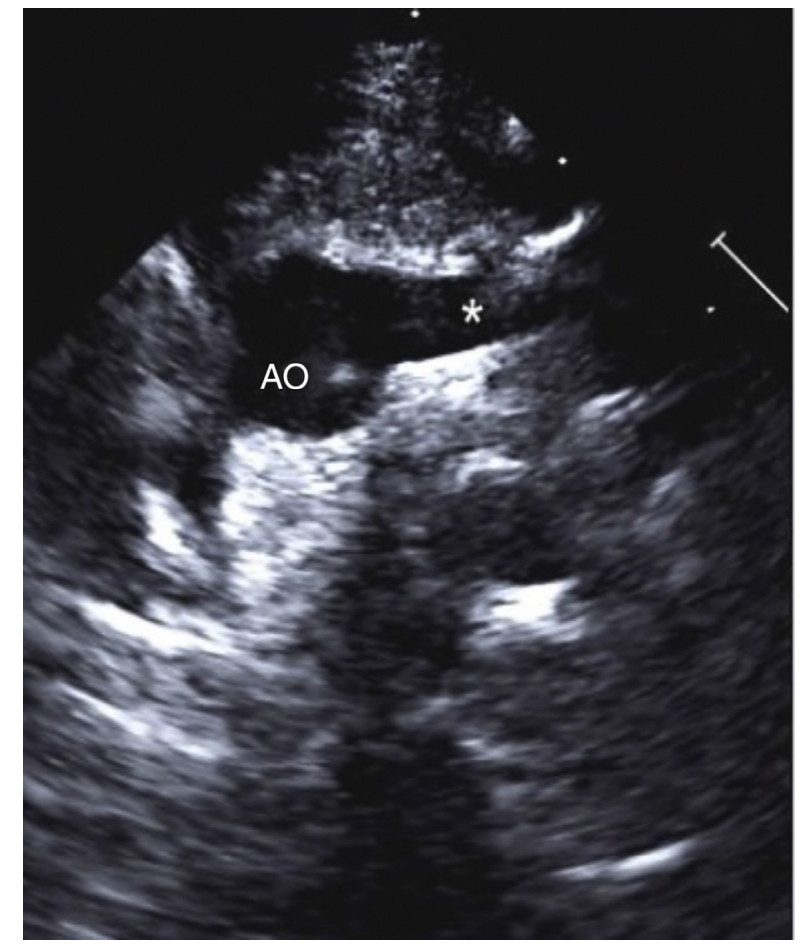

Figura 4 Ecocardiograma bidimensional desde la vista supraesternal en eje corto: se observa la aorta $(\mathrm{AO})$ y la emergencia del primer vaso supraaórtico $\left({ }^{*}\right)$ y su trayecto inicial hacia la izquierda.

\section{Conclusión}

Los anillos vasculares son una causa rara de cardiopatía congénita, los cuales deben sospecharse en el paciente pediátrico con síntomas respiratorios recurrentes o síntomas digestivos. En ausencia de manifestaciones clínicas, la presencia de un conducto arterioso contralateral al arco aórtico en los estudios de imagen debe indicar el diagnóstico.

\section{Financiación}

Ninguna.

\section{Conflicto de intereses}

Los autores de este artículo declaran no tener conflicto de intereses.

\section{Agradecimientos}

A I. Héctor Torres Rodríguez y Armando F. Saldaña Sesma.

\section{Bibliografía}

1. Cina CS, Althani H, Pasenau J, et al. Kommerell's diverticulum and right-sided aortic arch: A cohorte study and review of the literature. J Vasc Surg. 2004;39:131-9.

2. Yang C, Shu C, Li M, et al. Aberrant subclavian artery pathologies and Kommerell's diverticulum: A review and analisis of publish endovascular/hybrid treatment options. J Endovasc Ther. 2012;19:373-82.

3. Van Bogerijen GH, Patel HJ, Eliason JL, et al. Evolution in the management of aberrant subclavian arteries and related Kommerell diverticulum. Ann Thorac Surg. 2015;100: 47-53.

4. Idress J, Keshavamurthy S, Subramanian S, et al. Hybrid repair of Kommerell diverticulum. J Thorac Cardiovasc Surg. 2014;147:973-6.

5. Backer CL, Mavroudis C. Congenital Heart Surgery Nomenclature Database Project: Vascular rings, tracheal stenosis, pectus excavatum. Ann Thorac Surg. 2000;69:S308-18.

6. Backer CL, Mavroudis C, Rigsby CK, et al. Trends in vascular ring surgery. J Thorac Cardiovasc Surg. 2005;129: 1339-47.

7. Stone WM, Ricotta JJ 2nd, Fowl RJ, et al. Contemporary management of aberrant right subclavian arteries. Ann Vasc Surg. 2011;25:508-14.

8. Sierra-Galan LM, Shveid-Gerson D, Gomez-Garza G, et al. Double incomplete aortic arch and Kommerell's diverticulum as a cause of chronic cough. Arch Cardiol Mex. 2015;85: 158-60.

9. van Son JA, Konstantinov IE. Burckhard F. Kommerell and Kommerell's diverticulum. Tex Heart Inst J. 2002;29:109-12.

10. Tanaka A, Milner R, Ota T. Kommerell's diverticulum in the current era: A comprehensive review. Gen Thorac Cardiovasc Surg. 2015;63:245-59.

11. Velasquez G, Nath PH, Castaneda-Zuniga WR, et al. Aberrant left subclavian artery in tetralogy of Fallot. Am J Cardiol. 1980;45:811-8.

12. Hernanz-Schulman M. Vascular rings: A practical approach to imaging diagnosis. Pediatr Radiol. 2005;35:961-79.

13. Kimura ET, Meléndez G, Mendizábal AL, et al. Uncommon congenital and acquired aortic diseases: Role of multidetector CT angiography. RadioGraphics. 2010;30:79-98.

14. Backer CL, Hillman N, Mavoudris C, et al. Resection of Kommerell's diverticulum and left subclavian artery transfer for recurrent symptoms after vascular ring division. Eur J Cardiothorac Surg. 2002;22:64-9. 